Submission ID: 43756

\title{
Migration of Seismic Data for Multi-Layered Fractured Geological Media
} Using Elastic Approach

V.I. Golubev* (Moscow Institute of Physics and Technology), O.Y. Voinov (Moscow Institute of Physics and Technology), I.B. Petrov (Moscow Institute of Physics and Technology)

\section{SUMMARY}

Seismic surey process is the most common technology for prospecting and exploration of oil and gas deposits around the world. It allows to reconstruct the structure of the subsurface are on the basis of day surface movements caused by vibrational or explosive sources, due to the reflection of volumetric seismic waves from geological heterogeneities. Two different problems of seismic survey can be distinguished: inversion and migration. Development of new approaches to the solution of the latest one is the goal of this research.

This article is devoted to the problem of seismic imaging of layered fractured geological media. Authors proposed a new approach to the subsurface area image creation based on the numerical solution of the linear elasticity system. Adjoint operator approach was used, direct and adjoint problems were solved numerically by the grid-characteristic method on hexahedral meshes. Developed method allows to use arbitrary background models, even fractured. It was successfully applied to the model of the multi-layered fractured geolgical medium. 
Построение миграционных изображений многослойных трещиноватых геологических сред в упругой постановке

В.И. Голубев* (МФТИ), О.Я. Войнов (МФТИ), И.Б. Петров (МФТИ, ФГУ ФНЦ НИИСИ РАН)

\section{Введение}

Сейсмическая разведка является самой распространённой технологией поиска и разведки месторождений нефти и газа во всём мире. Она позволяет восстановить структуру подповерхностного пространства на основе данных о колебаниях дневной поверхности, инициированных вибрационными или взрывными источниками, из-за отражения объёмных сейсмических волн от геологических неоднородностей. При этом можно выделить два различных класса задач сейсмической разведки:

1) восстановление пространственного распределения механических характеристик среды (задача инверсии);

2) восстановление положения границ раздела геологических сред в условиях известной фоновой модели среды (задача миграции).

Разработке новых подходов к решению последней из перечисленных задач и посвящено настоящее исследование.

Одними из первых работ, относящихся к теории построения миграционных изображений, по праву считаются классические труды J.F. Claerbout $[1,2]$. В дальнейшем был предложен интегральный метод Кирхгофа [3]. В работе [4] использовано приближение Борна для построения линеаризованного соотношения между параметрами модели и наблюдёнными на поверхности данными. Одним из наиболее распространённых методов сейчас является технология обратной временной миграции (RTM) [5]. Хорошо современное состояние данной области освящено в обзоре [6]. В последнее время предпринимаются значительные усилия для повышения точности миграционных изображений сложных геологических сред [7,8]. При этом преимущество использования упругой модели геологической среды вместо акустической модели заключается в корректном воспроизведении в том числе и объёмных поперечных сейсмических волн и поверхностных волн-помех, в связи с чем появляется возможность более точного восстановления границ раздела в сложных случаях, например, при их углах наклона близких к вертикальным. Отметим, однако, что в изученных в процессе литературного обзора работах фоновая модель среды используется, как правило, достаточно простая: однородная среда, слоистая среда, среда без трещин, но с заданным пространственным распределением акустических или упругих свойств.

Настоящая работа посвящена исследованию проблемы построения миграционных изображений слоистых трещиноватых геологических сред. Авторами предложен подход, позволяющий получать изображения подповерхностного пространства путём численного решения полной определяющей системы уравнений линейной упругости. При этом используется идеология присоединённого оператора, а прямые и сопряжённые задачи решаются численно сеточно-характеристическим методом $[9,10]$ на гексаэдральных расчётных сетках. Разработанный метод позволяет, например, использовать модели фоновой среды произвольной сложности, в том числе трещиноватые. Его работоспособность продемонстрирована на модели многослойной трещиноватой геологической среды.

\section{Математическая модель и численный метод}

Рассмотрим подробнее подход, позволяющий проводить компьютерное моделирование процесса распространения сейсмических волн в трещиноватых геологических средах. Динамическое состояние линейно-упругой среды описывается определяющей системой уравнений в частных производных вида 


$$
\begin{aligned}
& \rho \dot{v}_{i}=\nabla_{j} \sigma_{i j}, \\
& \dot{\sigma}_{i j}=q_{i j k l} \dot{\varepsilon}_{k l} .
\end{aligned}
$$

Здесь $\rho$ - плотность среды, $v_{i}$ - компоненты вектора скорости смещения, $\sigma_{i j}$ и $\varepsilon_{k l}-$ компоненты тензоров напряжений Коши и деформации, $\nabla_{j}-$ ковариантная производная по $j$-й координате. Вид компонент тензора 4-го порядка $q_{i j k l}$ определяется реологией среды. Для линейно-упругого изотропного случая они имеют вид

$$
q_{i j k l}=\lambda \delta_{i j} \delta_{k l}+\mu\left(\delta_{i k} \delta_{j l}+\delta_{i l} \delta_{j k}\right) .
$$

В этом соотношении, которое обобщает закон Гука, $\lambda$ и $\mu$ - параметры Ламе, а $\delta_{i j}$ - символ Кронекера. В настоящей работе численно решается данная определяющая система уравнений на гексаэдральных расчётных сетках сеточно-характеристическим методом. При этом на этапе построения сетки производится явное выделение границ неоднородностей - наборов узлов с каждого из бортов трещин. В виду того, что решение уравнений акустики внутри трещины чрезвычайно ресурсоёмко используется подход с постановкой корректных граничных условий, обеспечивающий совпадение сейсмических откликов от трещиноватых объектов [11]. В узлах на противоположных бортах “флюидонасыщенной трещины” совпадают нормальные к плоскости трещины компоненты скорости, нормальные к плоскости трещины компоненты силы компенсируют друг друга, а тангенциальные компоненты силы равны нулю.

Рассмотрим подробнее способ построения миграционного изображения среды по получаемым в процессе сейсмической разведки полевым данным. Близость приближенного решения $\boldsymbol{m}$ обратной задачи к истинной модели среды можно оценить с использованием функционала невязки в следующем виде:

$$
\chi(\boldsymbol{m})=\frac{1}{2} \sum_{r} \int\left\|\boldsymbol{s}\left(\boldsymbol{x}_{r}, t ; \boldsymbol{m}\right)-\boldsymbol{d}\left(\boldsymbol{x}_{r}, t\right)\right\|^{2} d t .
$$

Здесь $\boldsymbol{x}_{r}$ - положение $r$-го приёмника, $\boldsymbol{s}$ и $\boldsymbol{d}$ - синтетическая и полевая сейсмограммы, суммирование ведется по всем приёмникам. Для изотропной упругой среды вариация функционала невязки может быть вычислена с помощью следующего интеграла по всему пространству [12]:

$$
\delta \chi=\int\left(K_{\rho} \delta \ln \rho+K_{\kappa} \delta \ln \kappa+K_{\mu} \delta \ln \mu\right) d^{3} x,
$$

где $\rho$ - плотность среды, $\kappa$ и $\mu$ - модуль всестороннего сжатия и модуль сдвига, $K_{\alpha}$ - ядро параметра $\alpha$. В данной работе в качестве условия визуализации было использовано ядро плотности, которое определяется из выражения

$$
K_{\rho}(\boldsymbol{x})=-\rho(\boldsymbol{x}) \int \boldsymbol{v}^{\dagger}(\boldsymbol{x},-t) \boldsymbol{v}(\boldsymbol{x}, t) d t,
$$

Здесь $\boldsymbol{v}$ - скорость, $\boldsymbol{v}^{\dagger}$ - сопряженная величина, в выражении фигурирует плотность приближенного решения обратной задачи, также называемого фоновой моделью среды. Для получения сопряженного поля скоростей используется сопряженный источник - в правую часть определяющей системы уравнений добавляется величина

$$
\boldsymbol{f}^{\dagger}(\boldsymbol{x}, t)=\sum_{r}\left[\boldsymbol{s}\left(\boldsymbol{x}_{r},-t\right)-\boldsymbol{d}(\boldsymbol{x},-t)\right] \delta\left(\boldsymbol{x}-\boldsymbol{x}_{r}\right)
$$

Построение миграционного изображения производится в несколько этапов. Сначала для фоновой модели среды решается прямая задача с конфигурацией источников и приемников, соответствующей произведённым наблюдениям. В результате этого получаются синтетические поля и сейсмограммы. Последние вместе с полевыми сейсмограммами используются на втором этапе для моделирования сопряженного поля. Наконец синтетическое и сопряженное поля используются для расчета ядра плотности. 
Отметим, что использование сеточно-характеристического метода для расчёта сейсмического поля позволяет:

1) получить синтетические сейсмограммы, содержащие отклики от трещиноватых объектов, для верификации предложенного метода построения миграционного изображения;

2) использовать более сложные фоновые модели среды, в том числе изначально трещиноватые.

\section{Результаты численного моделирования}

В работе исследовалось распространение сейсмических волн в трещиноватой слоистой среде в двумерной постановке. За основу модели был принят шестислойный вмещающий массив, геометрические и упругие характеристики которого взяты из работы [13]. Дополнительно на глубине 1650 м располагались две флюидонасыщенные субвертикальные макротрещины протяжённостью 100 м, наклонённые на угол 10 градусов к вертикали. Расчетная сетка имела шаг 5 м и содержала примерно 1 миллион узлов. В качестве начального возмущения использовалась группа точечных источников, расположенных на дневной поверхности через каждые 10 м и создающих вертикальную нагрузку вида импульс Рикера с пиковой частотой 30 Гц. Сейсмометры располагались на дневной поверхности через каждые 10 м и фиксировали значения вертикальной и горизонтальной компонент скорости смещения с шагом 0.45 мс на протяжении $3.3 \mathrm{c}$.

На Рисунке 1 приведены миграционные изображения, полученные с использованием описанного в работе подхода. Границы раздела слоёв на них отсутствуют, поскольку фоновая модель была изначально задана слоистой. На изображении при этом отчётливо выделяются места расположения двух трещин (Рисунок 1a). Отметим, что угол наклона трещины влияет на её след в миграционном изображении, и, соответственно, её ориентация также может быть однозначно восстановлена. В дальнейшем правая трещина была добавлена в фоновую модель, и также рассчитано миграционное изображение (Рисунок 1б). В данном случае оно не содержит особенностей в месте расположения правой трещины, что подтверждает корректность работы метода и возможность использования трещиноватых фоновых моделей.

a)
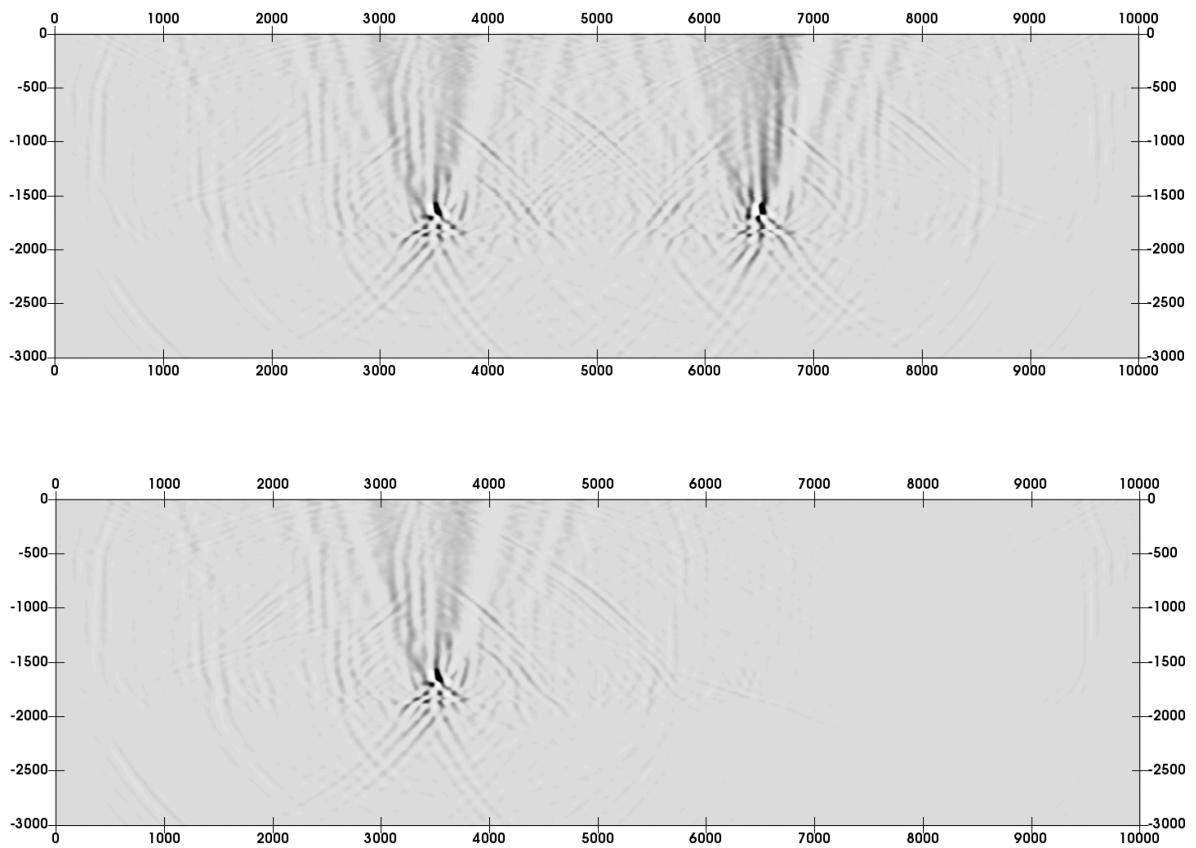

б)

Рисунок 1 Миграционное изображение слоистого геологического массива, содержащего две флюидонасыщенные субвертикальные трещины. В качестве фоновой среды использовалась слоистая модель без трещ̧ин (а) и с одной трещзиной (б). 


\section{Выводы}

В работе предложен алгоритм построения миграционных изображений трещиноватых геологических сред. Он основан на описании динамического поведения геологического массива системой уравнений линейной теории упругости. Её решение проводится численно сеточно-характеристическим методом на гексаэдральных расчётных сетках. В качестве условия визуализации выбран подход по вычислению ядра плотности. Отметим, что за счёт применения сеточно-характеристического метода для решения прямой и сопряжённой задачи появляется возможность использования более сложных фоновых моделей среды, в том числе трещиноватых. Предложенный подход был успешно применён для построения миграционного изображения многослойной трещиноватой среды в двумерной постановке. Направлением дальнейших исследований является изучение возможности его применения к геологическим средам, содержащим криволинейные геологические границы. В частности, планируется опробовать его на участке реального геологического массива, расположенного на северовостоке Европейской части Российской федерации, на территории Архангельской области, в пределах Ненецкого автономного округа, севернее Полярного круга, практически на берегу Баренцева моря [13].

\section{Благодарности}

Работа выполнена в рамках проекта РНФ №14-11-00263 на базе МФТИ.

\section{Библиография}

1. Claerbout, J.F. [1970] Coarse grid calculations of waves in inhomogeneous media with application to delineation of complicated seismic structure. Geophysics, 36(3), 407-418.

2. Claerbout, J.F., Doherty, S.M. [1972] Downward continuation of moveout-corrected seismograms. Geophysics, 37(5), 741-768.

3. French, W. S. [1975] Computer migration of oblique seismic reflection profiles. Geophysics, 40, 961-980.

4. Clayton, Robert W. and Stolt, Robert H. [1981] A Born-WKBJ inversion method for acoustic reflection data. Geophysics, 46(11), 1559-1567.

5. Baysal, E., D. Kosloff, and J. Sherwood [1983] Reverse time migration. Geophysics, 48, 15141524.

6. Etgen, J., Gray, S., and Zhang, Y. [2009] An overview of depth imaging in exploration geophysics. Geophysics, 74, WCA5-WCA17.

7. Jiao, K. et al. [2012] Elastic migration for improving salt and subsalt imaging and inversion. SEG Las Vegas Annual Meeting.

8. Voinov, O.Ya., Golubev, V.I., Petrov, I.B. [2016] Elastic imaging using multiprocessor computer systems. CEUR Workshop Proceedings, 1787, 491-495.

9. Golubev, V.I., Petrov, I.B., Khokhlov, N.I., Shul'ts, K.I. [2015] Numerical computation of wave propagation in fractured media by applying the grid-characteristic method on hexahedral meshes. Computational Mathematics and Mathematical Physics, 55(3), 509-518.

10. Golubev, V.I., Petrov, I.B., Khokhlov, N.I. [2015] Simulation of seismic processes inside the planet using the hybrid grid-characteristic method. Mathematical Models and Computer Simulations, 7(5), 439-445.

11. Левянт, В.Б., Миряха, В.А., Муратов, М.В., Петров, И.Б. [2015] Оценка влияния на сейсмический отклик степени раскрытости трещины и доли площади локальных контактов к ее поверхности. Технологии Сейсморазведки, 3, 16-30.

12. Luo Y., Tromp J., Denel B., Calandra H. [2013] 3D coupled acoustic-elastic migration with topography and bathymetry based on spectral-element and adjoint methods. Geophysics, 78(4), S193-S202.

13. Голубев, В.И., Петров, И.Б. [2016] Опыт расчёта сейсмических откликов от криволинейных геологических границ на основе их явного выделения. Технологии Сейсморазведки, 4, 45-50. 


\section{References}

1. Claerbout, J.F. [1970] Coarse grid calculations of waves in inhomogeneous media with application to delineation of complicated seismic structure. Geophysics, 36(3), 407-418.

2. Claerbout, J.F., Doherty, S.M. [1972] Downward continuation of moveout-corrected seismograms. Geophysics, 37(5), 741-768.

3. French, W. S. [1975] Computer migration of oblique seismic reflection profiles. Geophysics, 40, 961-980.

4. Clayton, Robert W. and Stolt, Robert H. [1981] A Born-WKBJ inversion method for acoustic reflection data. Geophysics, 46(11), 1559-1567.

5. Baysal, E., D. Kosloff, and J. Sherwood [1983] Reverse time migration. Geophysics, 48, 15141524.

6. Etgen, J., Gray, S., and Zhang, Y. [2009] An overview of depth imaging in exploration geophysics. Geophysics, 74, WCA5-WCA17.

7. Jiao, K. et al. [2012] Elastic migration for improving salt and subsalt imaging and inversion. SEG Las Vegas Annual Meeting.

8. Voinov, O.Ya., Golubev, V.I., Petrov, I.B. [2016] Elastic imaging using multiprocessor computer systems. CEUR Workshop Proceedings, 1787, 491-495.

9. Golubev, V.I., Petrov, I.B., Khokhlov, N.I., Shul'ts, K.I. [2015] Numerical computation of wave propagation in fractured media by applying the grid-characteristic method on hexahedral meshes. Computational Mathematics and Mathematical Physics, 55(3), 509-518.

10. Golubev, V.I., Petrov, I.B., Khokhlov, N.I. [2015] Simulation of seismic processes inside the planet using the hybrid grid-characteristic method. Mathematical Models and Computer Simulations, 7(5), 439-445.

11. Leviant, V.B., Miryakha, V.A., Muratov, M.V., Petrov, I.B. [2015] Seismic responses of vertical fractures depending on their thickness. Seismic Technologies, 3, 16-30.

12. Luo Y., Tromp J., Denel B., Calandra H. [2013] 3D coupled acoustic-elastic migration with topography and bathymetry based on spectral-element and adjoint methods. Geophysics, 78(4), S193-S202.

13. Golubev, V.I., Petrov, I.B. [2016] Experience of seismic responses from curvilinear geological boundaries modeling based on their explicit position description. Seismic Technologies, 4, 4550 . 